\section{BMJ Open Respiratory Research}

\title{
Effect of CPAP on cardiovascular events in minimally symptomatic OSA: long- term follow-up of the MOSAIC randomised controlled trial
}

\author{
Ivan Tang, ${ }^{1}$ Chris D Turnbull (1) , ${ }^{2,3}$ Dushendree Sen, ${ }^{2}$ Sonya Craig, ${ }^{4}$ \\ Malcolm Kohler, ${ }^{5}$ John R Stradling (i) ${ }^{3}$
}

To cite: Tang I, Turnbull CD, Sen D, et al. Effect of CPAP on cardiovascular events in minimally symptomatic OSA: long-term follow-up of the MOSAIC randomised controlled trial. BMJ Open Resp Res 2020;7:e000742. doi:10.1136/ bmjresp-2020-000742

IT and CDT are joint first authors.

Received 13 August 2020 Accepted 16 August 2020

\section{ABSTRACT}

The effect of continuous positive airway pressure (CPAP) on cardiovascular events is uncertain in minimally symptomatic obstructive sleep apnoea. Previous 2-year follow-up data from the Multicentre Obstructive Sleep Apnoea Intervention Cardiovascular (MOSAIC) trial showed a marginal reduction in cardiovascular events with CPAP therapy. We now present long-term MOSAIC study followup data. Median (first quartile, third quartile) follow-up was $5.0(2.2,5.0)$ and $3.7(1.5,5.0)$ years for CPAP and standard care, respectively. Compared to standard care, CPAP had no statistically significant effect on the risk of cardiovascular events $(\mathrm{HR}=0.83, \mathrm{p}=0.54,95 \% \mathrm{Cl}$ 0.46-1.51).
Check for updates

(C) Author(s) (or their employer(s)) 2020. Re-use permitted under CC BY-NC. No commercial re-use. See rights and permissions. Published by BMJ.

${ }^{1}$ Department of Anaesthesia and Intensive Care, Milton Keynes University Hospital, Milton Keynes, UK

${ }^{2}$ Oxford Centre for Respiratory Medicine, Oxford University Hospitals NHS Foundation Trust, Oxford, UK ${ }^{3} \mathrm{NIHR}$ Biomedical Research Centre Oxford, University of Oxford, Oxford, UK

${ }^{4}$ Liverpool Sleep and Ventilation Centre, University Hospital Aintree, Liverpool, UK

${ }^{5}$ Centre for Interdisciplinary Sleep Research, University of Zurich, Zurich, Switzerland

Correspondence to Dr Chris D Turnbull; christopher.turnbull@ouh. nhs.uk

\section{INTRODUCTION}

The Multicentre Obstructive Sleep Apnoea Intervention Cardiovascular (MOSAIC) trial was a multicentre, randomised controlled trial investigating the effect of continuous positive airway pressure (CPAP) treatment on calculated cardiovascular risk and daytime sleepiness in patients with minimally symptomatic obstructive sleep apnoea (OSA). Although CPAP improved daytime sleepiness, it did not improve calculated cardiovascular risk at 6 months. ${ }^{1}$

Two-year follow-up data of the Oxford patients found a statistically significant but marginal reduction (absolute reduction $9.6 \%, 95 \%$ CI $0 \%$ to $19 \%, \mathrm{p}=0.05)$ in new cardiovascular events in the CPAP group compared with standard care. ${ }^{2}$ This contrasts with the Sleep Apnoea Cardiovascular events (SAVE) Study which showed no improvement in new cardiovascular events following CPAP therapy. ${ }^{3}$

Despite evidence from observational studies, improvement in cardiovascular events resulting from CPAP in OSA remains unendorsed by randomised controlled trials. ${ }^{4}$

\section{METHODS}

We report long-term follow-up from all patients from a single centre of the MOSAIC trial. The MOSAIC trial was a multicentre, randomised controlled parallel, hospital trial in patients with confirmed OSA (oxygen desaturation index $>7.5$ /hour) but insufficient symptoms to mandate CPAP therapy. ${ }^{1}$

Patients were randomised to standard care or auto-adjusting CPAP therapy. The Oxford cohort was followed-up for a maximum of 5 years, and data were collected on new cardiovascular events. Data were analysed on an intention-to-treat basis. We prospectively recorded the occurrence of new cardiovascular events, including those leading to death. Cardiovascular events included angina, myocardial infarction, hypertension, peripheral vascular disease, atrial fibrillation, cardiac failure, stroke, transient ischaemic attack, coronary intervention and coronary artery bypass graft. Event-free survival was analysed using the log-rank test and Cox proportional HRs with multivariate analyses adjusted for age, baseline cardiovascular risk score and body mass index (BMI). A per protocol analysis was carried out of those patients who were randomised to CPAP who had usage of $>4$ hour $/$ night at last follow-up.

At 2-year follow-up 9\% and $4 \%$ in the standard care and CPAP arm groups experienced new events per year of follow-up. ${ }^{2}$ In order not to miss similar difference event rate over 5 years, with $80 \%$ power and two-sided alpha of $0.05,120$ patients were needed.

\section{RESULTS}

All 191 patients from a single centre are included in this follow-up analysis, with 94 initially randomised to the CPAP arm and 97 to the standard care arm. Patient characteristics are displayed in table 1 . Forty-three 
Table 1 Patient characteristics by treatment group. Data are displayed as mean \pm standard deviation, median (first quartile, third quartile) or number (\%)

\begin{tabular}{lccc}
\hline & CPAP & Standard care & $\begin{array}{c}\text { CPAP where usage } \\
\text { >4 hour/night }\end{array}$ \\
\hline Number & 94 & 97 & 25 \\
Baseline age (years) & $58.2 \pm 7.1$ & $57.7 \pm 7.6$ & $57.2 \pm 6.3$ \\
Male & $81(86 \%)$ & $84(87 \%)$ & $23(92 \%)$ \\
\hline Female & $13(14 \%)$ & $13(13 \%)$ & $2(8 \%)$ \\
\hline Baseline BMI (kg/m²) & $32.6 \pm 5.7$ & $32.4 \pm 5.3$ & $32.5 \pm 4.0$ \\
\hline ODI at presentation & $10.7(4.7,17.9)$ & $10.1(5.9,18.2)$ & $11.5(3.6,30.4)$ \\
\hline Baseline hypertension & $45(48 \%)$ & $47(49 \%)$ & $10(40 \%)$ \\
\hline Baseline diabetes mellitus & $15(16 \%)$ & $24(25 \%)$ & $3(12 \%)$ \\
Baseline atrial fibrillation & $4(4 \%)$ & $4(4 \%)$ & $1(4 \%)$ \\
\hline Baseline ischaemic heart disease & $11(12 \%)$ & $18(19 \%)$ & $2(8 \%)$ \\
Baseline cerebrovascular disease & $6(6 \%)$ & $2(2 \%)$ & $2(8 \%)$ \\
Current smoker & $10(11 \%)$ & $17(18 \%)$ & $24(17 \%)$ \\
\hline Length of follow-up (years) & $5.0(2.2,5.0)$ & $3.7(1.5,5.0)$ & $5.0(5.0,5.0)$ \\
\hline Number of crossovers & $43(46 \%)$ & $16(17 \%)$ & $0(0 \%)$ \\
\hline Numbers using CPAP at final follow-up & $38(40 \%)$ & $10(10 \%)$ & $25(100 \%)$ \\
\hline $\begin{array}{l}\text { Adherence in those using CPAP at final follow-up } \\
\text { (hours:min/night) }\end{array}$ & $5: 51(3: 22,6: 58)$ & $3: 32(1: 00,6: 56)$ & $6: 44(5: 51,7: 10)$ \\
\hline
\end{tabular}

BMI, body mass index; CPAP, continuous positive airway pressure; ODI, Oxygen desaturation index $>4 \%$.

patients (46\%) randomised to CPAP crossed over and stopped CPAP. Those randomised to CPAP and still using CPAP had good mean adherence, with median of mean usage of 5:51 hours:min/night (Interquartile range 3:226:58) at the end of the follow-up period; and 10 standard care subjects $(10 \%)$ had were using CPAP with median of mean usage of 3:32 hours:min/night (Interquartile range 1:00-6:55) by the end of the period.

There were 25 cardiovascular events in the CPAP group, compared with 32 in the standard care group. There was no significant difference in the univariate risk of cardiovascular events between CPAP and standard care $(\mathrm{HR}=0.83, \mathrm{p}=0.54,95 \% \mathrm{CI} 0.46$ to 1.51 , figure 1$)$. Multivariate adjustments for age, BMI and baseline cardiovascular risk score did not alter these results $(\mathrm{HR}=0.82$, $\mathrm{p}=0.52,95 \% \mathrm{CI} 0.45$ to 1.50$)$. A per protocol analysis of the risk of cardiovascular events in patients with CPAP usage $>4$ hour/night was also not significant, compared with standard care (HR=1.3, $\mathrm{p}=0.46,95 \%$ CI 0.61 to 2.94 ).

\section{DISCUSSION}

We have not shown CPAP to significantly impact cardiovascular event rates in minimally symptomatic OSA. This contrasts with our previous publication showing a reduction in cardiovascular events after 2 years of follow-up, ${ }^{2}$ hence the importance of reporting these longer term data. These results are in keeping with the much larger SAVE Study, ${ }^{3}$ which found that CPAP did not reduce cardiovascular events in patients screened for OSA following presentation to cardiovascular clinics.
Long-term follow-up in this study was limited by the small trial population, and thus wide confidence intervals of the estimates. However, given the marginal reduction in cardiovascular events observed at the 2 year follow-up, these data are important to report. The median length of follow-up was also longer in the CPAP arm as these patients were motivated to stay in touch to maintain their equipment. However, survival analyses take length of follow-up into account.

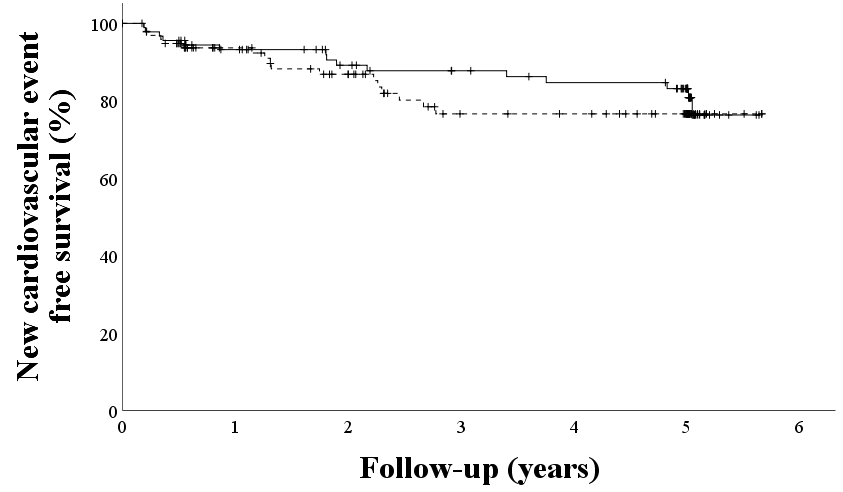

\begin{tabular}{lrlllll}
\multicolumn{5}{l}{ Number at risk } \\
& & & & & \\
CPAP & 94 & 76 & 64 & 58 & 54 & 43 \\
Standard & 97 & 70 & 56 & 39 & 37 & 25
\end{tabular}

Figure 1 Kaplan-Meier curve showing the percentage of patients without the occurrence of a cardiovascular event by the number of follow-up days for standard care (dotted line) or CPAP solid line. Log rank $p=0.54$. Cox proportional $\mathrm{HR}=0.83$ ( $p=0.54,95 \% \mathrm{Cl} 0.46$ to 1.51$)$. CPAP, continuous positive airway pressure. 
A theme consistent between MOSAIC, SAVE and the more recent Assessment of the Effect of CPAP on Energy and Vitality in Mild OSA (MERGE) trial, was the improvement of quality of life and symptoms with CPAP in even mild OSA. ${ }^{1-35}$ It is notable that the average usage of CPAP at the end of long-term follow-up, among those who did continue treatment was good ( $>5$ hour/night). This demonstrates that there are patients who adhere to CPAP in the long-term despite minimal symptoms. However, even among these individuals with good longterm adherence to CPAP there was no suggestion of a reduction in cardiovascular events.

Acknowledgements We would like to acknowledge support from the 0xford Health Services Research Committee and ResMed UK who supplied the CPAP machines for this trial. CT was funded and supported by an NIHR academic clinical lectureship. We would like to acknowledge the support of the National Institute for Health Research (NIHR) Oxford Biomedical Research Centre (BRC). The views expressed are those of the authors and not necessarily of the NHS, the NIHR or the Department of Health.

Contributors SC, MK and JRS conceived the initial trial and were responsible for trial design. IT and DS were responsible for data collection. IT and CDT were responsible for data analysis, literature searches and preparing the first draft of the manuscript. All authors viewed, commented and took responsibility for the final manuscript.

Funding The British Heart Foundation—unrestricted project grant.

Competing interests JRS has done paid consulting work for Bayer and Resmed, outside of the scope of this work. CDT has done paid consulting work for Bayer, outside of the scope of this work.MK reports personal fees from Bayer, personal fees from Astra, personal fees from Boehringer, grants from Roche, grants and personal fees from GSK, personal fees from Novartis, personal fees from Philips Respironics, outside the submitted work.

Patient consent for publication Not required.
Provenance and peer review Not commissioned; internally peer reviewed.

Data availability statement Data are available upon reasonable request including appropriate ethical approval. Qualified researchers may request access to patient level data and related study documents including the study protocol. Requests will be reviewed for scientific merit. Patient level data will be deidentified and study documents will be redacted to protect the privacy of trial participants. Please contact the corresponding author.

Open access This is an open access article distributed in accordance with the Creative Commons Attribution Non Commercial (CC BY-NC 4.0) license, which permits others to distribute, remix, adapt, build upon this work non-commercially, and license their derivative works on different terms, provided the original work is properly cited, appropriate credit is given, any changes made indicated, and the use is non-commercial. See: http://creativecommons.org/licenses/by-nc/4.0/.

ORCID iDs

Chris D Turnbull http://orcid.org/0000-0001-8942-5424

John R Stradling http://orcid.org/0000-0003-4971-5018

\section{REFERENCES}

1 Craig SE, Kohler M, Nicoll D, et al. Continuous positive airway pressure improves sleepiness but not calculated vascular risk in patients with minimally symptomatic obstructive sleep apnoea: the mosaic randomised controlled trial. Thorax 2012;67:1090-6.

2 Turnbull CD, Craig SE, Kohler M, et al. Cardiovascular event rates in the mosaic trial: 2-year follow-up data. Thorax 2014;69:950-2013204826.

3 McEvoy RD, Antic NA, Heeley E, et al. Cpap for prevention of cardiovascular events in obstructive sleep apnea. N Engl J Med 2016;375:919-31.

4 Peker Y, Balcan B. Cardiovascular outcomes of continuous positive airway pressure therapy for obstructive sleep apnea. $J$ Thorac Dis 2018;10:S4262-79.

5 Wimms AJ, Kelly JL, Turnbull CD, et al. Continuous positive airway pressure versus standard care for the treatment of people with mild obstructive sleep apnoea (merge): a multicentre, randomised controlled trial. Lancet Respir Med 2020;8:349-58. 